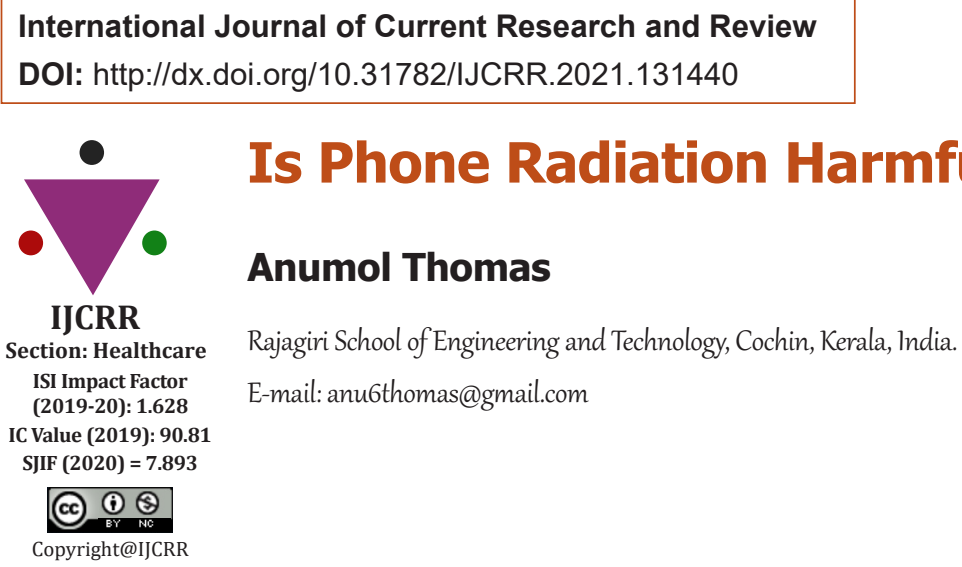

IJCRR

Section: Healthcare

ISI Impact Factor

(2019-20): 1.628

IC Value (2019): 90.81

$\operatorname{SIIF}(\mathbf{2 0 2 0})=7.893$

Anumol Thomas

Rajagiri School of Engineering and Technology, Cochin, Kerala, India.

E-mail: anu6thomas@gmail.com

\title{
- Is Phone Radiation Harmful to Health?
}

\section{MOBILE PHONE RADIATION}

Mobile phones, which are capable of receiving and transmitting high-frequency signals, are an integral part of our lives today. There are two types of opinions about mobile radiation, namely that the radiation emitted by the mobile phones we carry in our bodies is harmful to health but such an argument is not correct. However, some studies have shown that prolonged exposure to mobile radiation can cause damage to various tissues in the body. In these cases, it is essential to know how much radiation our mobile phone emits. The study of how radiation emitted while a mobile phone is operating affects human health is of great importance. As of June 2009, there are 4.3 billion mobile phone users worldwide. The mobile phone is powered by the lowest wavelength electromagnetic waves (microwaves). Most communication devices emit the same amount of radiation in different amounts.

\section{WORLD HEALTH ORGANIZATION REVELATIONS}

On May 31, 2011, the World Health Organization (WHO) revealed that mobile phone radiation could cause cancer in humans after electromagnetic radio waves were ranked $2 \mathrm{~B}$ in the International Cancer Research Center's (IARC) cancer list. The meeting, which included 31 experts from 14 countries, was held in Lyon, France, for eight days. Two of the longest-running studies in the last decade have found that those who use mobile phones the most are more likely. 13000 people participated in the experiment. People who use mobile phones more often have a $40 \%$ higher risk of developing glioma, a cancer that affects the brain, ie those who use 30 minutes a day for 10 years have not yet been able to determine whether mobile phone radiation can cause other cancers. The World Health Organization (WHO) has confirmed in its report that the 2-B classification as carcinogenic is logical. The WHO recommends using a speaker or headset to reduce mobile phone radiation until the study reports change, avoiding speech and relying on textmessages, thus minimizing processor proximity to the brain or using a landline phone.

\section{SPECIFIC ABSORPTION RATE (SAR)}

SAR is a measure of the radiation intensity of a mobile phone. (Specific Absorption Rate), i.e. SAR is the amount of radio frequency or high-frequency energy that our body absorbs when we use a mobile phone. Indicates. This measure is standardized by the Federal Communications Commission (FCC). SAR - the unit of measurement is watts/kilogram. According to FCC rules, the maximum SAR value that a mobile phone can emit is 1.6 watts $/ \mathrm{kg}$. The FCC says the lower it is, the safer it is. In addition, the FCC recommends that mobile phone users be warned of the dangers of radiation when purchasing a new phone. There are currently two types of SAR values offered by smartphone manufacturers. SAR (Head) value and SAR (Body) value; these two specific values refer to the amount of radio frequency energy emitted by the head and body parts of the human body. On most phones, this value is the only one the customer can verify. Low SAR for wireless devices, including cell phones. The value must be ensured. You can know the SAR value by dialing \# 07 \# from your smartphone. On some Android models that do not know this, you can enter the settings and understand the SAR value from the About Information section in the Legal Information section. SAR indicates the device in accordance with FCC standards. The value is not recognized as such in India. Thus, the maximum SAR in India is determined by taking into account the amount of radiation emitted from mobile phone towers from time to time. The Ministry of Telecommunications, Communications and IT has set up the Telecommunication Engineering Center (TEC) for this purpose. Determining and updating standards. As on September 1,2013 , the maximum SAR in India is 1.6 watts $/ \mathrm{kg}$ as per FCC standard. The previously allowed SAR was 2 watts $/ \mathrm{kg}$.

\section{REFERENCES}

1. "Market Data Summary (Q2 2009)". GSM Association.

2. "World Health Organization/International Agency for Research on Cancer Classifies Radiofrequency Electromagnetic Fields as Possibly Carcinogenic to Humans" (PDF). World Health Organization.

3. "World Health Organization: Cell Phones May Cause Cancer". Business Insider.

4. Hachman, Mark (31 May 2011). "WHO Finds Tentative Link Between Cell Phones, Cancer". pcmag.com. 